\title{
Effects of Acute Aerobic Exercise on Executive Function in Children With and Without Learning Disability: A Randomized Controlled Trial
}

\author{
Chung-Ju Huang
}

University of Taipei

Ming-Chun Hsueh

University of Taipei

Mei-Yao Huang

National Taiwan Sport University
Hsin-Yu Tu

National Ilan University

Yi-Hsiang Chiu

Chinese Culture University

Chien-Chih Chou

University of Taipei

This study examined the effects of acute aerobic exercise on sustained attention and discriminatory ability of children with and without learning disabilities (LD). Fifty-one children with LD and 49 typically developing children were randomly assigned to exercise or control groups. The participants in the exercise groups performed a 30-min session of moderate-intensity aerobic exercise, whereas the control groups watched a running/exercise-related video. Neuropsychological tasks, the Daueraufmerksamkeit sustained attention test, and the determination tests were assessed before and after each treatment. Exercise significantly benefited performance in sustained attention and discriminatory ability, particularly in higher accuracy rate and shorter reaction time. In addition, the LD exercise group demonstrated greater improvement than the typically developing exercise group. The findings suggest that the acute aerobic exercise influenced the sustained attention and the discriminatory function in children with LD by enhancing regulation of mental states and allocation of attentional resources.

Keywords: cognitive benefits, determination, sustained attention

A learning disability (LD) is defined as a neurobiological disorder of cognitive and/or language processing caused by atypical brain functioning (Silver et al., 2008).

C.-J. Huang, Hsueh, and Chou are with the Graduate Inst. of Sport Pedagogy, University of Taipei, Taipei City, Taiwan. Tu is with the Sports Education Center, National Ilan University, Yilan City, Taiwan. Chiu is with the Dept. of Physical Education, Chinese Culture University, Taipei City, Taiwan. M.-Y. Huang is with the Dept. of Physical Education Promotion, National Taiwan Sport University, Taoyuan City, Taiwan. Chou (ccc4453@gmail.com) is corresponding author. 
From a neuropsychological perspective, the manner in which individuals with LDs process and acquire information is different from the typical functioning expected for a child who can learn without great difficulty (Sharfi \& Rosenblum, 2016; Silver et al., 2008; Zhang, Fu, Xue, Zhao, \& Wang, 2019). Therefore, individuals with LDs may demonstrate inaccurate or slow and effortful reading, poor writing that lacks clarity, difficulties remembering numerical facts, and inaccurate mathematical reasoning (Silver et al., 2008). Particularly, children with LD generally have frontal cortex dysfunction (Krause \& Kadosh, 2013), which may compromise their executive functions (EFs) that determine the planning, selection, and ongoing regulation of behavior (Chan, Shum, Toulopoulou, \& Chen, 2008). Frequently, LDs are associated with weakness in EF domains, such as working memory (Moll, Göbel, Gooch, Landerl, \& Snowling, 2016), set shifting (Sharfi \& Rosenblum, 2016), sustained attention (Ebert \& Kohnert, 2011), discriminatory ability (Geary, Hoard, Nugent, \& Bailey, 2012), interference control (Palladino \& Ferrari, 2013), and response inhibition (van der Sluis, de Jong, \& van der Leij, 2004).

Among these EF domains, more attention should be paid to difficulties in sustained attention (Ebert \& Kohnert, 2011) and discriminatory ability (Geary et al., 2012) in children with LD. Sustained attention refers to the ability to direct and focus cognitive activity on specific stimuli and enables the maintenance of vigilance, selective and focused attention, response persistence, and continuous effort despite changing conditions (Cohen, 2011). A meta-analysis has supported the existence of sustained attention deficits in children with language impairment (Ebert \& Kohnert, 2011), as revealed by reduced accuracy compared with typically developing (TD) peers. Thus, the presence of sustained attention deficits among children with language difficulties raises the possibility that poor task or academic performance may be driven at least in part by sustained attention. Furthermore, discriminatory ability refers to a central component of EF to distinguish and determine multiple stimuli reactions in variables, which provide an understanding of reactive stress tolerance, attention deficits, and reaction speed in the presence of rapidly changing and continuous stimuli (Shmygalev et al., 2011). Previous research has also indicated that developmental dyslexia is associated with problems in perceptual anchoring utilized to establish the stimulus-specific predictions necessary to accurately perform tasks like frequency discrimination (Ahissar, 2007). Therefore, an impairment of the normal developmental trajectory for reading acquisition in children with reading difficulties could be associated with a weaker discriminatory ability. For children with LD, deficits in their sustained attention and discriminatory ability are important issues that should receive more attention. In addition to clinical treatments, alternative treatments such as physical exercise should be considered necessary to improve sustained attention and discriminatory ability in children with LD.

Given that children with LD are known to be less physically active than TD children (Cook, Li, \& Heinrich, 2015; Heller, Fisher, Marks, \& Hsieh, 2014), it is thought that exercise interventions may be of greater benefit, in terms of their impact on EF, to children with LD than to their more active, TD peers (Cook et al., 2015; Heller et al., 2014). In particular, the effects of a single bout of exercise have raised researchers' attention. A meta-analytic review has indicated that engaging in acute exercise could help children with neurodevelopmental disorders (e.g., intellectual and developmental disorders) enhance their EF performance, 
including areas such as inhibitory control, working memory, and cognitive flexibility (Soga, Kamijo, \& Masaki, 2016). For TD children, previous studies have revealed that acute exercise shows beneficial effects on vitality, reduced impulsivity, behavioral inhibition, cognitive development, and EF (Chang, Labban, Gapin, \& Etnier, 2012; Palmer, Miller, \& Robinson, 2013; Piepmeier et al., 2015). Especially, Palmer et al. (2013) found that preschoolers exhibited a markedly better ability to sustain attention after engaging in a bout of exercise, relative to after being sedentary. Hillman, Kamijo, and Pontifex (2012) also reported that single acute bouts of moderate-intensity exercise could be beneficial to neuroelectric indices reflecting the allocation of attentional resources in the service of stimulus discrimination. Furthermore, while the prefrontal cortices linked to the EF of children with LD may be abnormal in some respects (Kawashima et al., 2005), a single bout of exercise may activate the prefrontal brain regions related to better EF performance in some special populations of children (Best, 2010; Chang et al., 2012; Soga et al., 2016; Tomporowski, Davis, Lambourne, Gregoski, \& Tkacz, 2008). Even though the existing literature has illustrated a positive relationship between acute exercise and cognition in TD children, the effects of acute exercise on the sustained attention and discriminatory ability of children with LD have not been examined yet.

In light of the existing findings that children with LD exhibit relatively weak sustained attention and discriminatory ability, that these two EFs are positively affected by acute exercise, and that acute exercise, EF, and children with LD are potentially linked via various mechanisms (Ploughman, 2008), the hypothesis that acute exercise could improve the sustained attention and discriminatory ability in children with LD seems plausible. To the best of our knowledge, however, no studies have yet been conducted that have utilized neuropsychological tasks to investigate the impacts of acute exercise on sustained attention and discriminatory ability in children with LD. We theorized that the sustained attention and discriminatory ability of children with LD could be positively affected by acute exercise. Therefore, the purpose of this study was to further our understanding of the extent to which moderate-intensity exercise impacts the sustained attention and discriminatory ability of children with LD relative to their TD peers. We hypothesized that children participating in acute aerobic exercise would demonstrate greater positive changes in sustained attention and discriminatory ability compared with those not participating in the treatment. We also hypothesized that children with LD participating in acute exercise would demonstrate greater changes in those behavioral indices as compared with TD children participating in acute exercise. We believe that the results of this study could potentially be of critical importance in providing a basis for both further research in this area and the clinical application of exercise interventions in children with LD.

\section{Methods}

\section{Participants}

A total of 115 children (66 LD and 49 TD) aged between 10 and 13 years were recruited from eight primary schools located in northern Taipei, Taiwan. The TD children were recruited to confirm the severity of LD of our participants and further differentiate the cognitive effects of acute exercise on children with LD and TD 
children. In Taiwan, children with LD at the elementary school level are typically diagnosed (often in the second, third, or fourth grade) using the Diagnostic and Statistical Manual of Mental Disorders (4th ed.; American Psychiatric Association, 1994) criteria, consistent with the regulations and laws in Taiwan regarding special education. These criteria include (a) the presence of a verbal and/ or performance intelligence quotient (IQ) a score in the average range or above, (b) average scores of major academic achievement (in Chinese and mathematics) within the fifth to 10th percentile of the class, and (c) evidence of a processing deficit in one or more cognitive or linguistic and/or mathematical domains. Children with LD have been identified, via psychoeducational evaluations, as demonstrating LD in reading, writing, and/or mathematics. Most difficulties in reading, writing, and mathematics at the elementary school stage are reflected in slower reading, slower writing, and struggles with calculations relative to their peers, as well as attention difficulties. Those children identified as having LD have demonstrated evident academic learning difficulties (e.g., reading, writing, and/or mathematics impairments) and have been evaluated as being 2 years behind the TD children. Moreover, 15 children with LD were subsequently excluded because their individual school files stated that they were also diagnosed with Autism Spectrum Disorders or Attention Deficit Hyperactivity Disorders.

The final study sample was composed of 51 children with LD (29 boys and 22 girls) with a mean age of $12.02 \pm 0.81$ years (range $11-13$ ) and 49 aged-matched TD peers ( 23 boys and 26 girls) with a mean age of $12.12 \pm 0.78$ years (range $11-$ 13). Based on the information provided in their individual school files, the mean IQ of the children with LDs was $90.71 \pm 5.45$ (range 81-102), and the mean IQ of the TD children was $101.06 \pm 3.58$ (range 93-110). The TD children's grade level was appropriate to their age. For the purpose of this study, all the children with LD or TD children were randomly assigned into either the exercise groups ( $n=25$ for LD, $n=24$ for TD) or control groups ( $n=26$ for LD, $n=25$ for TD). The parents provided informed consent for their children's participation, and all procedures were in accordance with the ethical standards of the Office of Research and Development, University of Taipei.

\section{Measures}

Sustained Attention Test. The Daueraufmerksamkeit (DAUF) sustained attention test of the Vienna Testing System is an assessment of long-term selective attention and concentration. The sustained aspect of the test highlights the fact that attention operations become more difficult when they need to be continuously repeated. In this test, the participants watched rows of triangles that appeared against a black background on a computer screen, with the rows of triangles being presented under time-critical conditions. The tips of each triangle could point either up or down. In the S2 form of the test, a row of five triangles with either an upright or upside-down orientation appear on the monitor screen at irregular time intervals. The participants must then react only when two of the triangles point downward. During the test phase, 15 series of lines with five triangles were presented on a computer screen, and the participants had to press a button every time two of the five triangles pointed downward. We simply asked the participants to respond as quickly as possible without making mistakes. The total number of correct reactions 
to critical stimuli was recorded. A reaction was scored as "correct" if the reaction button was pressed while the critical stimulus was displayed and before the next stimulus was presented. For the DAUF performance, the mean correct response time was the average reaction time for stimuli that were reacted to correctly, and the number of correct trials was utilized to calculate the accuracy rate. In this study, the S2 form was used because it is recommended for use only with clients whose attentional performance is thought to be impaired (Schuhfried, 2010). The time required for this form was about $20 \mathrm{~min}$, including the instruction and practice phase.

Determination Test. The determination test was used to measure reactive stress tolerance and the associated ability to react. The test required the participant to use various cognitive skills to distinguish among different colors and sounds; to memorize the relevant characteristics of stimulus configurations, response buttons, and assignment rules; and to select the relevant responses according to the assignment rules laid down in the instructions and/or learned in the course of the test. The difficulty of the test arises from the need to sustain continuous, rapid, and varying responses to rapidly changing stimuli (Shmygalev et al., 2011). In this study, the participant's task was to react as quickly as possible to visual or acoustic stimuli by pressing the corresponding buttons on the response panel. There were five visual stimuli, colored white, yellow, red, green, and blue, which appeared in an upper and a lower row. The reaction buttons assigned to these five colors were arranged on the response panel in such a way that the respondent could use both hands. There were two additional visual stimuli, in the form of white, rectangular, visually distinct fields that appeared in the bottom left-hand and right-hand corners of the screen, to which the respondent had to react by pressing the corresponding (left or right) foot pedal. Two acoustic stimuli (a high tone and a low tone) were assigned to the two "sound" buttons in the middle of the panel. The lower, rectangular black button was pressed for a low tone, and the upper rectangular gray button was pressed for a high tone. The visual stimuli were presented on the screen, whereas the acoustic stimuli were presented via headphones. The duration of the stimulus presentation depended on the respondent's mean reaction time for the previous eight trials. If the response to a stimulus was not correct, the reaction time was doubled for the purpose of calculating the duration of the next stimulus. This test contained 180 trials, with 20 trials for each stimulus. The number of correct trials was utilized to calculate the accuracy rate, and the reaction time of each correct response was reported. The duration for the test was approximately $10 \mathrm{~min}$ for each participant. In terms of the reliability of Form S2, internal consistencies of between $r=.98$ and $r=.99$ have been reported for the main variables of accuracy rate and reaction time (Schuhfried, 2010).

\section{Baseline Demographic Information}

Physical fitness, academic achievement, and IQ were used as objective measures of baseline to ensure homogeneity in potential confounds between the four groups.

Physical Fitness. The measures of flexibility (sit and reach test), muscular endurance (sit-ups in $1 \mathrm{~min}$ ), power (standing long jump), and cardiovascular 
fitness (a half-mile run in the fastest possible time) were included for the fitness assessment. In addition to weight and height, each participant's physical fitness was estimated using the following procedure. The scores of the four subsets of the fitness assessment for all the participants were converted into standardized $T$ scores, and the overall physical fitness score was computed as the mean of the scores on these fitness subsets.

Academic Achievement. The language and mathematics tests were utilized in this study. The Chinese language test included reading comprehension passages with multiple-choice and open-ended questions, including prompts to acquire and read the printed words to uncover facts and ideas, find contextual relationships, understand word formation and syntax, make generalizations, and interpret information. The mathematics test consisted strictly of multiple-choice and open-ended questions regarding number series, with problems involving geometry, number operations, and algebra for fifth-, sixth-, and seventh-grade students. Standardized $T$ scores were used to convert the raw scores for the two tests, and the scores were computed as the mean of the scores on these language and mathematics tests.

Intelligence Quotient. The IQ score of each participant, which was evaluated by the Wechsler Intelligence Scale for Children-IV when he/she was in the fourth grade, was collected in this study. The Chinese version of the Wechsler Intelligence Scale for Children-IV, which is the most popular and widely researched test of children's intelligence, is a collection of 13 distinct subtests divided into two scales, namely, a Verbal Scale and a Performance Scale. The test has good reliability and validity (Wechsler, 1991), and its subtest reliabilities are moderate to excellent $(0.61-0.92)$.

\section{Exercise Intensity}

Heart rate (HR) reserve was used as a method to establish exercise intensity in this study. For each participant in the two exercise groups, the HR reserve was calculated as the maximal HR minus resting HR, where "206.9- $(0.67 \times$ age $)$ " was an indirect formula for estimating the maximal HR. During the bout of exercise, the desired target HR of the main exercise stage was of moderate to vigorous intensity (50$70 \%$ ), which is suggested by the American College of Sports Medicine (2010) exercise guidelines. This moderate-to-vigorous exercise intensity has been known to have beneficial effects on EF (Chang et al., 2012; Tomporowski, 2003). In this study, the target HR was calculated by a formula as follows: target $\mathrm{HR}=$ (maximal $\mathrm{HR}$ - resting $\mathrm{HR}) \times$ percentage intensity desired + resting $\mathrm{HR}$. To determine each participant's resting HR, the measurement was performed with the participant in a supine position in our lab after a 10- to 15 -min period without any physical activity. Also, each participant was asked verbally about their quality of sleep the preceding night and their mental stress level to ensure that the measurement was only performed when they were healthy and feeling well.

Each participant completed the rating of perceived exertion (RPE), which was defined as their perception of their own level of effort during the exercise (Borg, 1998). The RPE rates a participant's perceived exertion on a scale of 6 (very, very light to fairly light exertion) to 20 (maximal exertion). The RPE was recorded at 2-min intervals during the main exercise stage. 


\section{Procedures}

The experimental procedure included four stages: (a) confirmation, (b) pretest, (c) treatment, and (d) posttest. During the confirmation stage, which took place during the fall semester in 2017, each participant and their parent visited the school and were asked to read and complete the consent form and a medical history questionnaire. Then, the height and weight of each participant was measured. The language and mathematics tests were administered to each participant in a random order. It took $40 \mathrm{~min}$. After a 20 -min break, the fitness test was conducted under the supervision of our trained research assistants. All the participants were asked not to engage in intense physical activity on the day of their visit.

Upon the second visit on another day, each participant then entered the pretest stage, during which, the sustained attention and determination tests were administered in a random order. For the DAUF sustained attention test, the participant was verbally instructed on how to respond as quickly as possible when the expected number of upside-down triangles was shown. Then, the participants would have approximately $5 \mathrm{~min}$ for practice before the formal test. After a 5-min rest in a quiet classroom, the given participant was instructed on how to perform the determination test and had 24 practice trials. Subsequently, the formal test was conducted. For each practice trial, the experimenter provided feedback by saying either "correct" or "incorrect," and no other feedback or instruction was provided.

During the treatment stage, the exercise groups performed a single bout of aerobic exercise. The participants were instructed to run on a treadmill (Cybex; Rosemont, IL) for $30 \mathrm{~min}$. The exercise directions called for a warm-up period lasting $5 \mathrm{~min}$, a main exercise period lasting $20 \mathrm{~min}$, and a cooldown period lasting $5 \mathrm{~min}$. The exercise intensity of the main exercise period was set at $50-70 \%$ of each participant's individual HR reserve. During the main exercise period, each participant was required to report his or her RPE every $2 \mathrm{~min}$. The speed of the treadmill was adjusted as necessary, based on the target HR and the RPE. A Polar watch (Polar RS800CX; Polar Electro Oy, Kempele, Finland) was worn to measure each participant's HR during the entire exercise stage, with the HR data from the HR monitor being recorded at 1-min intervals. Three HR variables were identified, with HR-pre and HR-post representing the HRs assessed before and immediately after the single bout of exercise, respectively, and HR-average representing the average $\mathrm{HR}$ assessed during the main exercise period. In contrast, we simply asked the participants in the control groups to watch a running/exercise-related video for 30 min during the treatment stage. The video was produced by the experimenter for the purpose of maintaining a calm state during the control procedure. The content of the video consisted of information about the benefits of exercise on health, with some of the clips within the video being presented as cartoons to make them intriguing for the children.

In the posttest stage, which began within 5-10 min of the end of treatment, we asked each participant again to complete the determination test and the DAUF sustained attention test using the same directions and under the same conditions as were used during the pretest. The entire experimental process lasted $90 \mathrm{~min}$. A week following the completion of the stages, detailed explanations of the fitness test and academic tests used in this study were provided. 


\section{Statistical Analysis}

The effects of exercise manipulation on HR was examined using a 2 (group: LD and TD) $\times 3$ (time: HR-pre, HR-average, and HR-post) mixed analysis of variance (ANOVA). In addition, 2 (group: $\mathrm{LD}$ and TD) $\times 2$ (treatment: exercise and control) $\times 2$ (time: pretest and posttest) mixed ANOVAs were used to determine the effects of acute exercise on performance of the DAUF and the determination tests for the children with LD and the TD children. An analytical comparison of the effects of exercise between the children with LD and TD children was conducted through independent $t$ tests, as indicated by the differences in accuracy rates and reaction time of the DAUF and determination tests from preperformance and postperformance tests. Small to moderate effect sizes (ESs) were observed for each of the EF domains of interest $(0.2=$ small ES, $0.5=$ moderate ES, and $0.8=$ high ES). The ES was calculated according to Cohen's $d$ (which is equal to the mean difference of the groups divided by the pooled $S D)$, and a partial eta-squared $\left(\eta_{\mathrm{p}}^{2}\right)$ was reported for the significant main effect and interaction. All statistical analyses were conducted in SPSS (version 19.0; IBM, Armonk, NY), with $\alpha$ equal to .05.

\section{Results}

\section{Demographic Analyses}

To ensure homogeneity in the potential confounds between the four groups, oneway ANOVAs or $\chi^{2}$ tests were used to compare the demographic data between the groups. The analyses indicated that no differences were found between the four groups in terms of age, height, weight, body mass index, cardiovascular fitness, muscular strength, muscular endurance, or flexibility, $F(3,96)=0.18,0.47,0.34$, $0.08,0.82,1.77,0.95$, and 0.92 , all $p s>.05$. In addition, no differences were found in gender and grade, $\chi^{2}=1.01$ and 0.18 , all $p s>.05$, respectively. However, the TD children reported significantly higher scores than the children with LD on the academic tests of language and mathematics, as well as in terms of IQ, $F(3,96)=$ 23.72, 64.79, and 41.86, all $p s<.05$, respectively. No differences in the academic tests and IQ were found between the TD exercise and TD control groups, as well as between the LD exercise and LD control groups. Detailed descriptive data are summarized in Table 1.

\section{Exercise Manipulation Check}

The descriptive data of HR for exercise are summarized in Table 2. A 2 (group) $\times 3$ (time) mixed ANOVA revealed a significant main effect of Time, $F(2,47)=$ $4472.99, p<.0001, \eta_{p}^{2}=.99$, indicating an increase in HR from the HR-pre $(73.80$ \pm 1.83 beats per minute) to the HR-average ( $159.88 \pm 6.14$ beats per minute), and a decrease in HR from the HR-average to the HR-post $(104.14 \pm 3.33$ beats per minute) across the LD and TD exercise groups. In brief, the exercise groups showed that the single bout of exercise induced arousal and that this arousal was slightly reduced after they ceased exercising. Furthermore, no main effect of Group, $F(1,48)=0.61, p>.05$, and interaction of Group $\times$ Time, $F(2,47)=0.03$, $p>.05$ were observed. During the main exercise period, there were no differences 


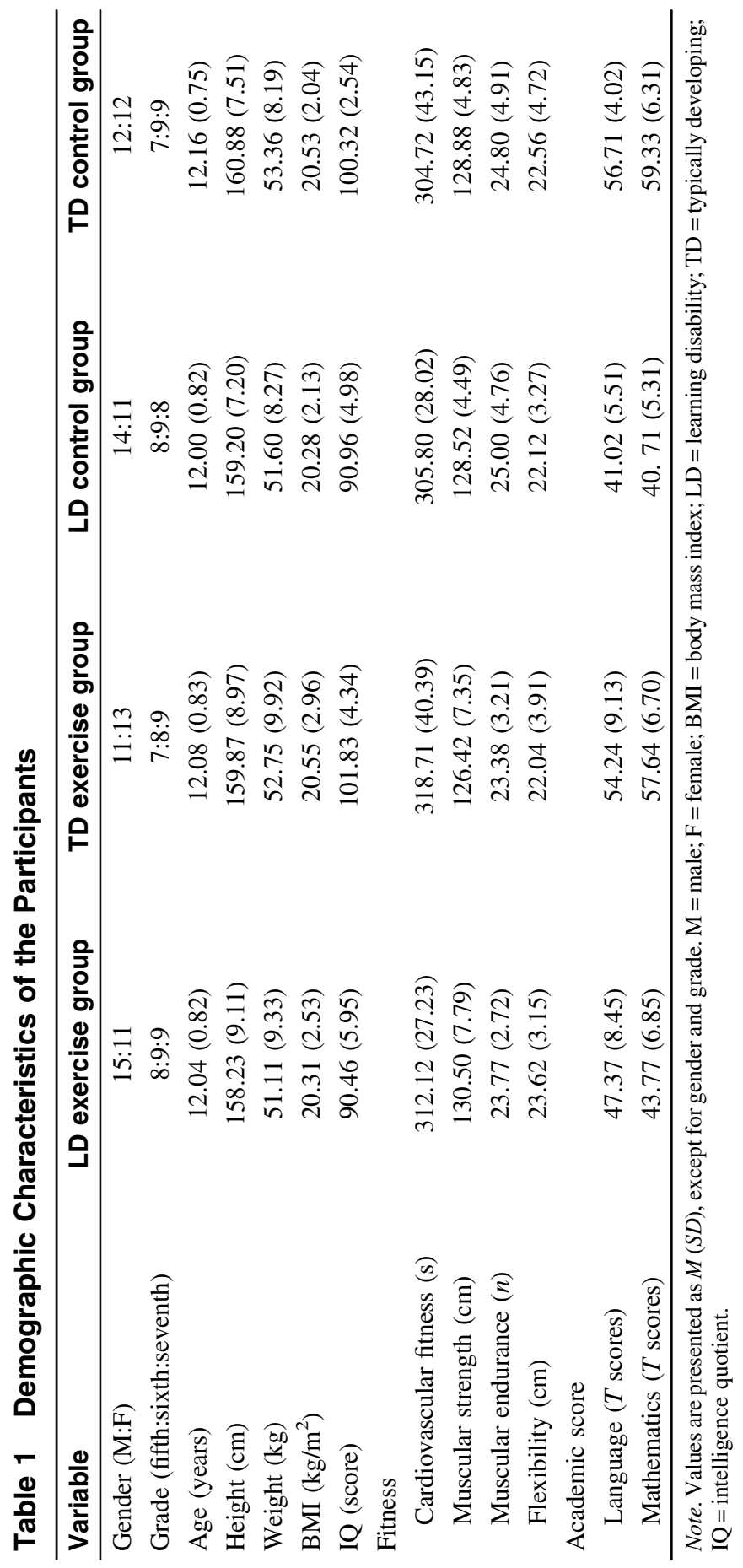




\section{Table 2 Descriptive Data of HR for Exercise Manipulation Check}

\begin{tabular}{lcccc}
\hline Variables & $\begin{array}{c}\text { LD exercise } \\
\text { group }\end{array}$ & $\begin{array}{c}\text { TD exercise } \\
\text { group }\end{array}$ & $\begin{array}{c}\text { LD control } \\
\text { group }\end{array}$ & $\begin{array}{c}\text { TD control } \\
\text { group }\end{array}$ \\
\hline HR-pre (bpm) & $74.12(2.10)$ & $73.46(1.44)$ & $74.44(1.78)$ & $74.40(2.00)$ \\
HR-average & $160.19(7.29)$ & $159.54(4.76)$ & $75.52(1.05)$ & $75.56(1.85)$ \\
(bpm) & & & & $75.80(2.10)$ \\
HR-post (bpm) & $104.35(4.06)$ & $103.92(2.36)$ & $74.92(1.89)$ & - \\
RPE (scale) & $16.04(1.28)$ & $16.67(1.05)$ & - & - \\
$\begin{array}{l}\text { Treadmill speed } \\
\text { (km/hr) }\end{array}$ & $6.72(0.50)$ & $6.65(0.25)$ & - & - \\
\hline
\end{tabular}

Note. Values are presented as $M(S D) . \mathrm{HR}=$ heart rate; $\mathrm{LD}=$ learning disability; TD = typically developing; bpm = beats per minute; $\mathrm{RPE}=$ rating of perceived exertion; HR-pre $=\mathrm{HR}$ assessed before the single bout of exercise; HR-post $=H R$ assessed immediately after the single bout of exercise; HR-average $=$ the average $\mathrm{HR}$ assessed during the main exercise period.

in the RPE or running speed between the LD and TD exercise groups, $t(48)=-1.89$ and .62 all $p s>.05$.

\section{Normality on Neuropsychological Test Variables}

Based on the discussion of normal or symmetrical distribution provided by Kellar and Kelvin (2012), we determined that the absolute value of skewness of the measures in both tests of sustained attention and discriminatory ability fell between -0.41 and 0.52 for the accuracy rate and between .08 and .86 for the mean correct response time or reaction time. For the absolute value of kurtosis, the measures in both tests fell between -1.20 and -0.05 for the accuracy rate and between 0.45 and 1.32 for the mean correct response time or reaction time. The results of the data could be regarded as normal or symmetrically distributed.

\section{Sustained Attention}

As indicated by the accuracy rates for the DAUF test presented in Figure 1, the mixed ANOVA showed significant main effects of Treatment, $F(1,96)=34.58$, $p<.001, \eta_{\mathrm{p}}^{2}=.27$, Group, $F(1,96)=22.50, p<.001, \eta_{\mathrm{p}}^{2}=.19$, and Time, $F(1,96)$ $=226.80, p<.001, \eta_{\mathrm{p}}^{2}=.70$. Importantly, a three-way interaction of Treatment $\times$ Group $\times$ Time was observed, $F(1,96)=56.49, p<.05, \eta_{\mathrm{p}}^{2}=.37$. Further decomposition revealed that significant interactions of Treatment $\times$ Time were observed for both the LD group, $F(1,49)=285.36, p<.001, \eta_{\mathrm{p}}^{2}=.85$, and the TD group, $F(1,47)=7.63, p<.01, \eta_{\mathrm{p}}^{2}=.14$. For the LD and TD groups, the participants who received the exercise treatment had a greater accuracy rate at the posttest than the pretest (all $p s<.001$ ), with high ES (4.04 and 1.34). In contrast, for both the LD group and the TD group, those who received the control treatment exhibited no difference in the accuracy rate between the pretest and the posttest $(p=.134$ and .154). In addition, the TD children exhibited a higher accuracy rate than the LD children in the pretest, $t(98)=-7.51, p<.0001$.

With respect to the mean correct response time shown in Figure 1, the mixed ANOVA indicated significant main effects of Treatment, $F(1,96)=11.08, p<.01$, 


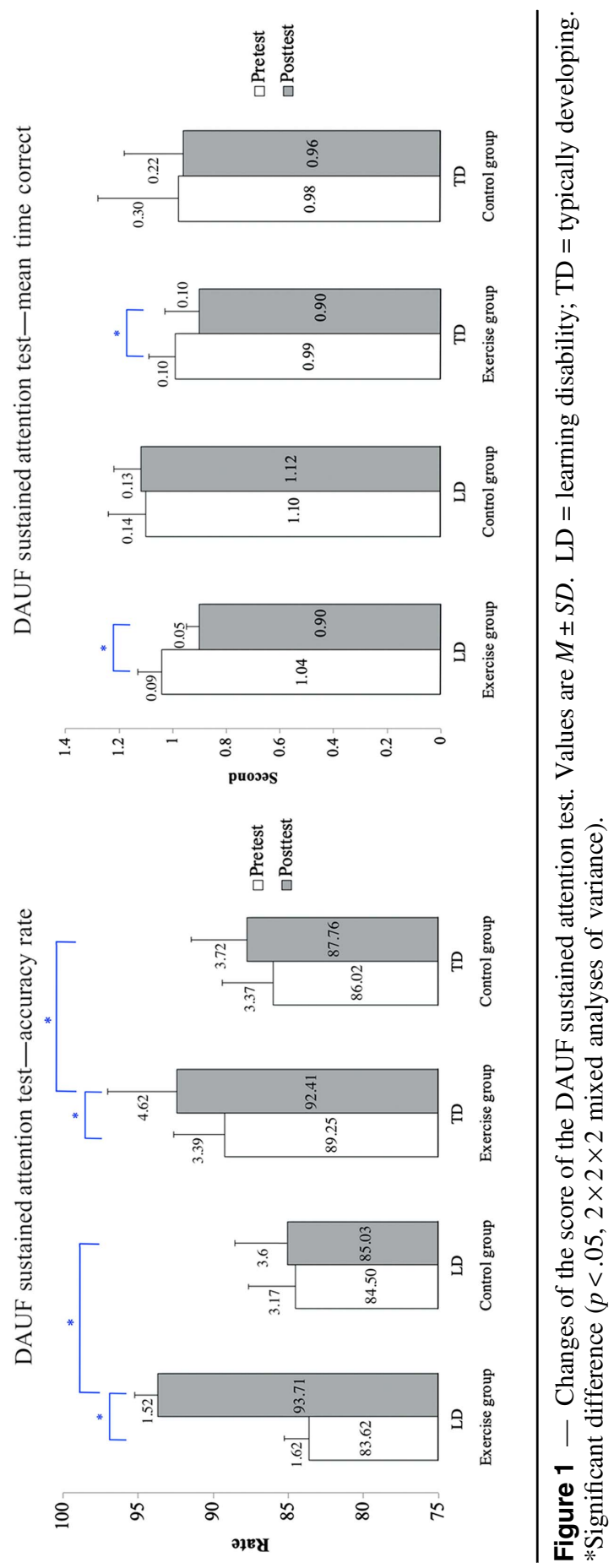


$\eta_{\mathrm{p}}^{2}=.10$, Group, $F(1,96)=11.04, p<.001, \eta_{\mathrm{p}}^{2}=.10$, and Time, $F(1,96)=8.30$, $p<.01, \eta_{\mathrm{p}}^{2}=.08$. In addition, the analysis showed significant interactions of Treatment $\times$ Time, $F(1,96)=8.55, p<.01, \eta_{\mathrm{p}}^{2}=.08$ and Treatment $\times$ Group, $F(1,96)=4.69, p<.05, \eta_{\mathrm{p}}^{2}=.05$. Further decomposition revealed that the participants who received the exercise treatment had a faster mean correct response time at the posttest than the pretest, $t(49)=10.58, p<.001$, regardless of the group with high ES (ES = 1.51). However, no difference in the mean correct response time was found between the pretest and the posttest for the participants who received the control treatment across the groups, $t(49)=-.02, p>.05$. Regardless of the group, the participants who received the exercise treatment had a faster mean correct response time than those who received the control treatment at the posttest. No significant simple main effects were found within the interaction of Treatment $\times$ Group. In addition, the TD children exhibited a higher accuracy rate than the LD children in the pretest, $t(98)=2.30, p<.05$.

Regarding comparisons of changes (posttest minus pretest) in performance at the DAUF test between the LD and TD exercise groups, the results revealed a greater change in the accuracy rate for the LD exercise group $\left(M_{\text {change }}=10.10 \%\right)$ than the TD exercise group $\left(M_{\text {change }}=3.10 \%\right), t(48)=10.52, p<.01$, and a larger change in the mean correct response time for the LD exercise group $\left(M_{\text {change }}=-0.14 \mathrm{~s}\right)$ than the TD exercise group $\left(M_{\text {change }}=-0.09 \mathrm{~s}\right), t(48)=-2.26, p=.029$.

\section{Determination Ability}

As indicated by the accuracy rates for the determination test presented in Figure 2, the mixed ANOVA showed significant main effects of Treatment, $F(1,96)=$ $19.62, p<.001, \eta_{\mathrm{p}}^{2}=.17$, Group, $F(1,96)=9.93, p<.01, \eta_{\mathrm{p}}^{2}=.09$, and Time, $F(1$, $96)=49.84, p<.001, \eta_{\mathrm{p}}^{2}=.34$. In addition, the analysis revealed significant interactions of Treatment $\times$ Time, $F(1,96)=25.15, p<.01, \eta_{\mathrm{p}}^{2}=.21$, and Group $\times$ Time, $F(1,96)=7.29, p<.001, \eta_{\mathrm{p}}^{2}=.07$. No interaction of Treatment $\times$ Time $\times$ Group was observed, $F(1,96)=0.61, p>.05$. Given the purpose of the study, only the interactions related to the treatment were further examined. Further decomposition indicated that the participants who received the exercise treatment had a higher accuracy rate at the posttest than the pretest, $t(49)=-10.72, p<.001$, regardless of the group with high ES $(\mathrm{ES}=1.53)$. However, no difference in the accuracy rate was found in either group between the pretest and the posttest for the participants who had received the control treatment, $t(49)=1.19, p>.05$. Regardless of the group, the participants who received the exercise treatment had a higher accuracy rate than those who received the control treatment at the posttest. However, the TD children had a significantly higher accuracy rate than the LD children in the pretest, $t(98)=-4.23, p<.01$.

With respect to the reaction time results shown in Figure 2, the mixed ANOVA showed significant the main effects of Treatment, $F(1,96)=4.27, p<.05, \eta_{\mathrm{p}}^{2}=.04$, Group, $F(1,96)=8.87, p<.01, \eta_{\mathrm{p}}^{2}=.09$, and Time, $F(1,96)=46.51, p<.001$, $\eta_{\mathrm{p}}^{2}=.33$. In addition, the analysis revealed significant interactions of Treatment $\times$ Time, $F(1,96)=21.24, p<.01, \eta_{\mathrm{p}}^{2}=.18$, and Group $\times$ Time, $F(1,96)=5.37, p<$ $.05, \eta_{\mathrm{p}}^{2}=.05$. No interaction of Treatment $\times$ Time $\times$ Group was observed, $F(1,96)=$ $2.58, p>.05$. Further decomposition indicated that the participants who received the exercise treatment exhibited a faster reaction time at the posttest than the 


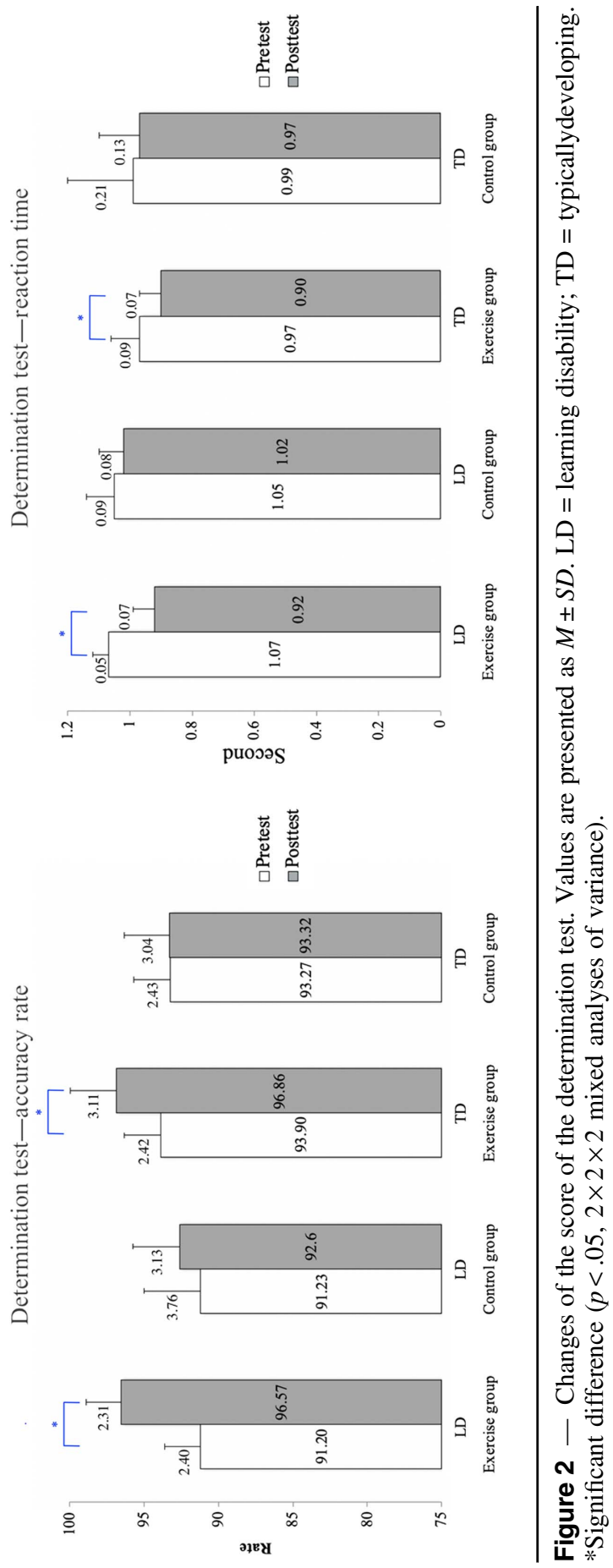


pretest, $t(49)=9.69, p<.001$, regardless of the group with high ES (ES = 1.37). However, no difference in the reaction time was found in either group between the pretest and the posttest for the participants who had received the control treatment, $t(49)=1.32, p>.05$. Regardless of the group, the participants who received the exercise treatment had a faster reaction time than those who received the control treatment at the posttest. However, the TD children had a significant faster reaction time than the children with LD in the pretest, $t(98)=3.27, p<.01$.

Regarding comparisons of changes in performance at the determination test between the LD and TD exercise groups, the results revealed a greater change in the accuracy rate for the LD exercise group $\left(M_{\text {change }}=5.37 \%\right)$ than the TD exercise group $\left(M_{\text {change }}=2.97 \%\right), t(48)=3.36, p=.002$, and a larger change in the reaction time for the LD exercise group $\left(M_{\text {change }}=-0.15 \mathrm{~s}\right)$ than the TD exercise group $\left(\mathrm{M}_{\text {change }}=-0.07 \mathrm{~s}\right), t(48)=-3.67, p=.001$.

\section{Discussion}

The present study examined the effect of an acute bout of aerobic exercise on sustained attention and discrimination ability in children with LD. With the intensity of exercise confirmed via HR and RPE monitoring, the results demonstrated that a single session of aerobic exercise led to shortened reaction times and improved accuracy rates on the DUAF sustained attention test and the determination test, even as the LD and TD control groups, which did not undergo the exercise session, showed no such improvements. The results provided strong evidence of acute exercise on children's performance in tasks requiring sustained attention or discriminatory ability, with a high ES (25\% of variance explained; Cohen, 1988, p. 287). Also, compared with the TD children who participated in the exercise treatment, the children with LD participating in acute exercise demonstrated greater positive impact in the reaction times and accuracy rates. Our findings suggest that higher accuracy rate and fast reaction time could be improved in response to a short bout of exercise. The findings also indicated that the effect was greater on children with LD than TD children.

Consistent with our hypothesis, the results indicated that children who received the exercise treatment had shortened reaction times and improved accuracy rates on the DAUF sustained attention test compared with children who received control treatment at the posttest, and they also exhibited significant improvements after the acute aerobic exercise. The results are consistent with the previous literature by Pontifex, Parks, Henning, and Kamijo (2015), that acute exercise appears to induce enhanced attentional processing. Given that aerobic exercise typically involves engagement in various forms of physical activity in a rhythmic manner and strengthens the cardiovascular system, a single acute bout of aerobic exercise could positively regulate mental states (e.g., mood, self-control, self-efficacy, and concentration) to energize attention resources to refine cognitive processing (Basso \& Suzuki, 2017; Tomporowski, 2003). Moreover, current evidence suggests that the brain regions linked to the function of sustained attention are activated after acute exercise (Palmer et al., 2013). It may be that acute exercise has a positive impact on subsequent tasks requiring sustained attention via an influential activation of the dorsolateral prefrontal cortex. Notably, 
when the intensity of acute aerobic exercise goes to the moderate levels, sustained attention would be improved via increased cognitive resources (i.e., the attention and effort) during aerobic exercise, while elevated arousal levels are improved during the postexercise period. However, although the known impact of acute exercise on sustained attention is limited, more research is needed to further explore its potential effects.

Similarly, children in the exercise groups not only exhibited shortened reaction times and higher accuracy rates in the discrimination task compared with the control groups, but also have significant improvements from the pretest to the posttest. The results indicated that acute aerobic exercise enhances attention function relevant to the discriminatory ability in children, consistent with the existing literature (Hillman et al., 2012; Pontifex et al., 2015). These two studies indicated that acute exercise is beneficial to the efficiency of attentional resource allocation. Thus, raised arousal levels related to the cardiovascular system during the moderate-vigorous intensity of aerobic exercise may be associated with increased efficiency in attentional allocation after exercise. Using a neuroelectrical approach, previous research has also observed the effects of running induced improvements in the medial prefrontal cortex and orbitofrontal cortex (Brockett, LaMarca, \& Gould, 2015). The results pattern is consistent with our behavioral data, demonstrating that acute aerobic exercise could enhance performance on the allocation of attentional resources and neuroprocessing efficiency, resulting in the improved ability to distinguish and identify multiple stimuli reactions in variables. That is, the findings of this study provide support where a single bout of aerobic exercise may enhance the efficiency of attentional processing. Although the present study did not include any physiological and neuroelectric measures during or after the acute exercise, clearly, this is an area that warrants further attention to better understand how acute aerobic exercise might influence EF acquisition in a physical activity setting.

Nonetheless, when considered as a whole, the findings of previous studies indicate that a single bout of exercise induces arousal levels that activate brain processes and that biochemical factors may contribute to the regulation of cognitive function (Chang et al., 2012). The mechanisms underlying these benefits may involve complex neurochemical changes and modified functioning of brain areas within the limbic circuit. For instance, acute aerobic exercise was previously found to be associated with decreased cortisol and increased brain-derived neurotrophic factor (BDNF), serotonin, and dopamine (Chang et al., 2012). These biological mechanisms contribute to attention processing and inhibition in terms of regulating arousal levels in frontostriatal-frontocerebellar circuits and enhancing the control of EF (Del Campo, Chamberlain, Sahakian, \& Robbins, 2011). In animal studies, increased BDNF levels in the hippocampus after exercise were found to be related to elevated EF (Palencia \& Ragozzino, 2005; Vaynman, Ying, \& Gomez-Pinilla, 2004). Although further studies are still required to confirm these findings, previous studies suggest that acute aerobic exercise may result in improved cognitive function in children due to the alteration of neurochemical expressions.

Although the pretest sustained attention and discrimination ability were different between children with LD and TD children, the children with LD participating in the acute exercise exhibited greater positive changes in those behavioral indices compared with the TD children participating in the acute exercise. With sufficient statistical power, this study supported that acute exercise 
might demonstrate greater effects on the sustained attention and discrimination ability in children with LD than TD children. The results are consistent with the previous literature regarding the effects of physical exercise on the attentionrelated symptoms of children with different disabilities (Piepmeier et al., 2015; Soga et al., 2016; Vogt, Schneider, Abeln, Anneken, \& Strüder, 2012). Given that children with LD are likely to be associated with cognitive dysfunction, they may gain greater cognitive benefits from the acute exercise than TD children because the effects induced by the exercise would distinctly compensate for the deficit in EF (Cook et al., 2015; Heller et al., 2014). Alternatively, TD children would also receive cognitive benefits from a single acute bout of exercise; however, these benefits might be limited due to their cognitive function exhibiting a ceiling effect that could not have a capacity to be developed.

Although this study indicated that acute aerobic exercise could enhance sustained attention and discrimination ability among the children with LD and TD children, some limitations warrant caution and can be considered in future research. First, although the four groups have shown homogeneities in gender, age, body mass index, and physical fitness, the findings of this study might be attributed to small sample sizes and other confounders. For example, several confounders, such as physical activity amount, sleep quality, emotion, memory, perception, and even motor skills, have been found to covary with EFs. However, this study did not take these variables into consideration. Second, the different types of LD, as well as time spent playing video games, using the Internet, and watching television, may confound the effects of acute aerobic exercise on EF in children with LD. As such, further research is required to rule out the influences of these factors. Finally, future research may investigate and expand upon the present findings by reporting the level of physical activity outside the exercise intervention and applying cognitive and behavioral perspectives to the assessment of LD.

\section{Conclusions}

This study revealed the effects of acute exercise on the sustained attention and discrimination ability in children with and without LD. Importantly, the benefits were significantly greater for children with LD than their TD peers. Tentative explanations for the effects of exercise in children with LD could be the influences on the regulation of mental states and allocation of attentional resources. Therefore, our positive findings are in accordance with those of previous studies demonstrating the beneficial effects of moderately intense aerobic exercise programs on sustained attention and discrimination ability in children with LD. Given the beneficial effects of aerobic exercise on certain cognitive functions in children with LD, schools and the parents of children with LD should consider maximizing the opportunities that such children have to engage in moderately intense structured aerobic exercises.

\section{Acknowledgments}

The research was supported in part by grants by the Ministry of Science and Technology, MOST 107-2410-H-845-023. 


\section{References}

Ahissar, M. (2007). Dyslexia and the anchoring-deficit hypothesis. Trends in Cognitive Sciences, 11, 458-465. PubMed ID: 17983834

American College of Sports Medicine. (2010). American college of sports medicine's guidelines for exercise testing and prescription (8th ed.). Philadelphia, PA: Lippincott Williams and Wilkins.

American Psychiatric Association. (1994). Diagnostic and statistical manual of mental disorders (4th ed.). Washington, DC: American Psychiatric Publishing.

Basso, J.C., \& Suzuki, W.A. (2017). The effects of acute exercise on mood, cognition, neurophysiology, and neurochemical pathways: A review. Brain Plasticity, 2(2), 127-152. PubMed ID: 29765853 doi:10.3233/BPL-160040

Best, J.R. (2010). Effects of physical activity on children's executive function: Contributions of experimental research on aerobic exercise. Developmental Review, 30(4), 331-351. PubMed ID: 21818169

Borg, G. (1998). Borg's perceived exertion and pain scales. Champaign, IL: Human Kinetics.

Brockett, A.T., LaMarca, E.A., \& Gould, E. (2015). Physical exercise enhances cognitive flexibility as well as astrocytic and synaptic markers in the medial prefrontal cortex. PLoS One, 10(5), e0124859. doi:10.1371/journal.pone.0124859

Chan, R.C., Shum, D., Toulopoulou, T., \& Chen, E.Y. (2008). Assessment of executive functions: Review of instruments and identification of critical issues. Archives of Clinical Neuropsychology, 23(2), 201-216. PubMed ID: 18096360 doi:10.1016/j.acn. 2007.08.010

Chang, Y.K., Labban, J.D., Gapin, J.I., \& Etnier, J.L. (2012). The effects of acute exercise on cognitive performance: A meta-analysis. Brain Research, 1453, 87-101. PubMed ID: 22480735

Cohen, J. (1988). Statistical power analysis for the behavioral sciences (2nd ed.). Hillsdale, NJ: Lawrence Erlbaum Associates.

Cohen, R.A. (2011). Sustained attention. In J.S. Kreutzer, J. DeLuca, \& B. Caplan (Eds.), Encyclopedia of clinical neuropsychology (pp. 282-292). New York, NY: Springer.

Cook, B.G., Li, D., \& Heinrich, K.M. (2015). Obesity, physical activity, and sedentary behavior of youth with learning disabilities and ADHD. Journal of Learning Disabilities, 48(6), 563-576. PubMed ID: 24449262 doi:10.1177/0022219413518582

Del Campo, N., Chamberlain, S.R., Sahakian, B.J., \& Robbins, T.W. (2011). The roles of dopamine and noradrenaline in the pathophysiology and treatment of attentiondeficit/ hyperactivity disorder. Biological Psychiatry, 69, e145-e157. PubMed ID: 21550021

Ebert, K.D., \& Kohnert, K. (2011). Sustained attention in children with primary language impairment: A meta-analysis. Journal of Speech, Language, and Hearing Research, 54(5), 1372-1384. PubMed ID: 21646419 doi:10.1044/1092-4388(2011/10-0231)

Geary, D.C., Hoard, M.K., Nugent, L., \& Bailey, D.H. (2012). Mathematical cognition deficits in children with learning disabilities and persistent low achievement: A fiveyear prospective study. Journal of Educational Psychology, 104(1), 206-223. PubMed ID: 27158154

Heller, T., Fisher, D., Marks, B., \& Hsieh, K. (2014). Interventions to promote health: Crossing networks of intellectual and developmental disabilities and aging. Disability and Health Journal, 7(Suppl. 1), S24-S32. doi:10.1016/j.dhjo.2013.06.001

Hillman, C.H., Kamijo, K., \& Pontifex, M.B. (2012). The relation of ERP indices of exercise to brain health and cognition. In H. Boecker, C.H. Hillman, L. Scheef, \& H.K. Struder (Eds.), Functional neuroimaging in exercise and sport sciences (pp. 419-446). New York, NY: Springer. doi:10.1007/978-1-4614-3293-7_18 
Kawashima, R., Okita, K., Yamazaki, R., Tajima, N., Yoshida, H., Taira, M., . . . Sugimoto, K. (2005). Reading aloud and arithmetic calculation improve frontal function of people with dementia. The Journals of Gerontology, Series A: Biological Sciences \& Medical Sciences, 60(3), 380-384. doi:10.1093/gerona/60.3.380

Kellar, S.P., \& Kelvin, E.A. (2012). Munro's statistical methods for health care research. Philadelphia, PA: Wolters Kluwer Health/Lippincott Williams and Wilkins.

Krause, B., \& Kadosh, R.C. (2013). Can transcranial electrical stimulation improve learning difficulties in atypical brain development? A future possibility for cognitive training. Developmental Cognitive Neuroscience, 6, 176-194. PubMed ID: 23770059 doi:10. 1016/j.den.2013.04.001

Moll, K., Göbel, S.M., Gooch, D., Landerl, K., \& Snowling, M.J. (2016). Cognitive risk factors for specific learning disorder: Processing speed, temporal processing, and working memory. Journal of Learning Disabilities, 49(3), 272-281. PubMed ID: 25124507 doi:10.1177/0022219414547221

Palencia, C.A., \& Ragozzino, M.E. (2005). The contribution of NMDA receptors in the dorsolateral striatum to egocentric response learning. Behavioral Neuroscience, 119(4), 953-960. PubMed ID: 16187823 doi:10.1037/0735-7044.119.4.953

Palladino, P., \& Ferrari, M. (2013). Interference control in working memory: Comparing groups of children with atypical development. Child Neuropsychology, 19(1), 37-54. PubMed ID: 22122432

Palmer, K.K., Miller, M.W., \& Robinson, L.E. (2013). Acute exercise enhances preschoolers' ability to sustain attention. Journal of Sport and Exercise Psychology, 35(4), 433-437. PubMed ID: 23966452

Piepmeier, A.T., Shih, C.H., Whedon, M., Williams, L.M., Davis, M.E., Henning, D.A., . . Etnier, J.L. (2015). The effect of acute exercise on cognitive performance in children with and without ADHD. Journal of Sport and Health Science, 4(1), 97-104.

Ploughman, M. (2008). Exercise is brain food: The effects of physical activity on cognitive function. Developmental Neurorehabilitation, 11(3), 236-240. PubMed ID: 18781504

Pontifex, M.B., Parks, A.C., Henning, D.A., \& Kamijo, K. (2015). Single bouts of exercise selectively sustain attentional processes. Psychophysiology, 52(5), 618-625. PubMed ID: 25523887 doi:10.1111/psyp.12395

Schuhfried, G. (2010). Vienna test system: Psychological diagnostic. Mödling, Austria: Schuhfried GmbH.

Sharfi, K., \& Rosenblum, S. (2016). Executive functions, time organization and quality of life among adults with learning disabilities. PLoS One, 11(12), e0166939. PubMed ID: 27959913

Shmygalev, S., Damm, M., Weckbecker, K., Berghaus, G., Petzke, F., \& Sabatowski, R. (2011). The impact of long-term maintenance treatment with buprenorphine on complex psychomotor and cognitive function. Drug and Alcohol Dependence, 117(2-3), 190-197.

Silver, C.H., Ruff, R.M., Iverson, G.L., Barth, J.T., Broshek, D.K., Bush, S.S., . . Planning, C. (2008). Learning disabilities: The need for neuropsychological evaluation. Archives of Clinical Neuropsychology, 23(2), 217-219. doi:10.1016/j.acn.2007.09.006

Soga, K., Kamijo, K., \& Masaki, H. (2016). Effects of acute exercise on executive function in children with and without neurodevelopmental disorders. The Journal of Physical Fitness and Sports Medicine, 5(1), 57-67.

Tomporowski, P.D. (2003). Cognitive and behavioral responses to acute exercise in youths: A review. Pediatric Exercise Science, 15(4), 348-359. doi:10.1123/pes.15.4.348

Tomporowski, P.D., Davis, C.L., Lambourne, K., Gregoski, M., \& Tkacz, J. (2008). Task switching in overweight children: Effects of acute exercise and age. Journal of Sport and Exercise Psychology, 30(5), 497-511. PubMed ID: 18971509 doi:10.1123/jsep. 30.5.497 
van der Sluis, S., de Jong, P.F., \& van der Leij, A. (2004). Inhibition and shifting in children with learning deficits in arithmetic and reading. Journal of Experimental Child Psychology, 87, 239-266. PubMed ID: 14972600

Vaynman, S., Ying, Z., \& Gomez-Pinilla, F. (2004). Hippocampal BDNF mediates the efficacy of exercise on synaptic plasticity and cognition. European Journal of Neuroscience, 20(10), 2580-2590. PubMed ID: 15548201 doi:10.1111/j.1460-9568. 2004.03720.x

Vogt, T., Schneider, S., Abeln, V., Anneken, V., \& Strüder, H. (2012). Exercise, mood and cognitive performance in intellectual disability-A neurophysiological approach. Behavioural Brain Research, 226(2), 473-480. PubMed ID: 22019342 doi:10. 1016/j.bbr.2011.10.015

Wechsler, D. (1991). Manual for the Wechsler Intelligence Scale for Children (4th ed.). San Antonio, TX: Psychological Corporation.

Zhang, X., Fu, W., Xue, L., Zhao, J., \& Wang, Z. (2019). Children with mathematical learning difficulties are sluggish in disengaging attention. Frontiers in Psychology, 10, 932. PubMed ID: 31143141 doi:10.3389/fpsyg.2019.00932 